\title{
Centrifugal compressor performance assessment under different impeller tip clearance sizes from far to near stall conditions
}

\author{
Reza Taghavi-Zenouz ${ }^{\mathrm{a}}$ And Ehsan Solki \\ School of Mechanical Engineering, Iran University of Science and Technology, Narmak, 16846-13114 Tehran, Iran
}

Received 8 August 2013, Accepted 20 May 2014

\begin{abstract}
Impeller tip clearance is a key parameter in design process of a centrifugal compressor which has influential effects on its aerodynamic and thermodynamic performances. Interaction of tip leakage flow with the main stream could be the major source for occurrence of stall phenomenon. In the present investigation, two impeller tip clearances of small and large sizes are investigated under different flow coefficients assigned to "design", "low flow" and "high flow" conditions. Flow field is precisely simulated utilizing solution of the Reynolds-Averaged Navier-Stokes equations. Influence of the impeller stall on the volute flow field is studied. Structure of vortical flows within the impeller passages and volute are demonstrated in details. Numerical results showed that the flow within the volute becomes stall-prone while the flow within the impeller stalls. In contrast to the high flow case, the low flow condition was more prone for the stall occurrence. Performance tests were also conducted on Iran University of Science and Technology (IUST) centrifugal compressor test rig for validation of the numerical results.
\end{abstract}

Key words: stall / centrifugal compressor / performance map / tip clearance

\section{Introduction}

Considering tip clearance in compressor blades is inevitable. These gaps are associated with flow instabilities, which may lead to poor thermo-dynamical and fluid-dynamical performances, when being inappropriately designed.

Rotating stall is one of the flow instability phenomenon in which a circumferentially uniform flow pattern is disturbed by local flow separation. At low flow rate, the compressor blades are subjected to an increased incidence angle which may cause local separation. This separation, or blockage, will restrict the flow through the blade passage which in turn causes a deflection of the incoming flow around the separated region. Local separation packets propagate from blade to blade in the direction of rotor rotation and are known as "rotating stall". This instability results in poor performance of the compressor and may lead to the fatigue damage of the blades.

For the last few decades, effects of tip leakage flow on occurrence of instabilities inside the centrifugal compressors, like stall and surge phenomena, are investigated by some researchers [1-6]. These studies reveal the fact that the interaction of the tip leakage flow with the incoming

${ }^{a}$ Corresponding author: taghavi@iust.ac.ir main passage flow is the major source for the stall onset. Most research works on the effects of the impeller tip clearance level on the compressor performance are carried out for a single operating condition, for example, for near stall condition, whereas, for a successful compressor design process, investigations should be extended to a wider operating range.

Three-dimensional flow fields in a backward impeller at design and off-design (choked, and close to surge) conditions have been studied experimentally and numerically by Hah and Krain [7]. In their numerical approach, they used a three-dimensional viscous flow solver to study the development of the secondary flows, vortex motion, and tip-clearance flow structure. Based on their experimental and numerical results, they concluded that the swirling flow and vortex motion inside the impeller are greatly influenced by the relative motion between the rotating impeller and the stationary casing as well as by the resulting tip leakage flow.

Chriss et al. [8], have studied impeller flow field of NASA Large Scale Centrifugal Compressor (LSCC) at its design point and lower mass flow rates conditions. They illustrated throughflow velocity magnitudes as well as the secondary flow structures inside the impeller utilizing laser Doppler velocimetry. They compared their results with those obtained through their numerical simulations, but have not investigated the effects of different 


\section{Nomenclature}

\begin{tabular}{|c|c|}
\hline$b_{2}$ & Impeller exit width $(\mathrm{mm})$ \\
\hline$C_{\mathrm{r} 2}$ & Radial velocity at impeller exit $\left(\mathrm{m} \cdot \mathrm{s}^{-1}\right)$ \\
\hline$C R$ & $\begin{array}{l}\text { Tip clearance ratio = tip clearance size/ } \\
\text { tip chord length }\end{array}$ \\
\hline$d_{2}$ & Impeller outlet diameter $(\mathrm{mm})$ \\
\hline$I$ & Unit tensor \\
\hline L.E. & Leading edge \\
\hline$N$ & Rotational speed $\left(\operatorname{rad} . \mathrm{s}^{-1}\right)$ \\
\hline$p$ & Static pressure $(\mathrm{Pa})$ \\
\hline P.S. & Pressure surface \\
\hline$r$ & Radial position \\
\hline S.S. & Suction surface \\
\hline T.E. & Trailing edge \\
\hline$U_{2}$ & $\begin{array}{l}\text { Peripheral velocity at impeller exit } \\
\left(\mathrm{m} . \mathrm{s}^{-1}\right)\end{array}$ \\
\hline$u_{\tau}$ & Friction velocity $\left(\mathrm{m} \cdot \mathrm{s}^{-1}\right)$ \\
\hline$Q$ & Volume flow rate $\left(\mathrm{m}^{3} \cdot \mathrm{s}^{-1}\right)$ \\
\hline$\vec{v}$ & Absolute velocity $\left(\mathrm{m} . \mathrm{s}^{-1}\right)$ \\
\hline$V_{\mathrm{r}}$ & Radial velocity component $\left(\mathrm{m} \cdot \mathrm{s}^{-1}\right)$ \\
\hline$\vec{v}_{\mathrm{r}}$ & Relative velocity $\left(\mathrm{m} \cdot \mathrm{s}^{-1}\right)$ \\
\hline$v_{\theta}$ & Tangential velocity $\left(\mathrm{m} \cdot \mathrm{s}^{-1}\right)$ \\
\hline$y^{+}$ & Non-dimensional wall distance \\
\hline$\Omega$ & Angular velocity $\left(\mathrm{rad}_{\mathrm{s}} \mathrm{s}^{-1}\right)$ \\
\hline$\overline{\bar{\tau}}$ & Stress tensor $(\mathrm{Pa})$ \\
\hline$\varphi$ & Flow coefficient \\
\hline$\psi$ & Loading factor \\
\hline$\nu$ & Kinematic viscosity $\left(\mathrm{m}^{2} \cdot \mathrm{s}^{-1}\right)$ \\
\hline$\theta$ & Azimuthal angle (degree) \\
\hline
\end{tabular}

tip clearance sizes of the impeller and flow conditions on the flow field in other components, like the volute.

Reunanen [9] numerically and experimentally has studied a radial compressor with three different volute cross sections under various flow conditions. He has examined different volute cross sections in order to find optimum stability and operating range of the compressor, and offered proposals for improvement of the compressor performance, especially at high mass flow conditions. Detailed measurements of swirling flow inside a centrifugal compressor volute with elliptical cross section have been carried out by Ayder et al. [10]. They showed that the flow inside the volute is highly three dimensional, and that the swirling velocity component has an important influence on the crosswise and circumferential variation of the static pressure and velocity distribution. In addition, they observed important variations of the secondary vortex structures at different volute cross sections.

Investigations on compressor performance under different impeller tip clearance sizes and at various flow coefficients can produce a wide domain of data which can be used for proper design of compressors. Most of the investigations are restricted to one component, either the impeller or the volute, and these two major parts are not studied simultaneously. The reality is that the performance of the compressor components is strongly coupled to each other and overall compressor instability cannot be determined by one component alone. The key question, which has not been properly answered yet, is that what happens in the volute flow field when the impeller stalls. So, in the present research work, flow characteristics in the impeller and volute of a centrifugal compressor are simultaneously studied under different operating conditions. Initially, since the tip leakage flow is the major reason for the stall onset, the performance of the impeller with different tip clearance sizes is investigated from far to near stall conditions. Then, the volute flow field is studied before and after the impeller stall, in order to find out that if it is stall-prone or not.

\section{Test model}

Test model was a centrifugal compressor equipped with a proper data logging system. Figure 1 shows IUST compressor test rig. The compressor impeller and volute together with the pressure taps are shown in Figure 2. The compressor incorporated in this test rig is a mechanically driven unit used for supercharging large vehicle engines. Its impeller of $145 \mathrm{~mm}$ in outer diameter and tip clearance size of $0.6 \mathrm{~mm}$ (for $C R=1.3 \%$ ) consists of 10 radialtipped blades. Geometric parameters of the compressor and the ambient conditions are presented in Table 1.

This compressor is driven by a $15 \mathrm{~kW}, 2950 \mathrm{rev} \cdot \mathrm{min}^{-1}$, AC, 3-phase electro-motor via a v-belt. There is a gearbox to multiply the impeller speed to 4.4 times the driving pulley speed. Compressor rotational speed was controlled by a variable frequency drive capable of changing electrical frequency from 0 to $50 \mathrm{~Hz}$.

The Reynolds number based on the impeller outlet diameter, $d_{2}$ (Fig. 3a) and the peripheral velocity at the impeller exit, $U_{2}$ (Fig. 3b) was about $6.2 \times 10^{5}$.

Compressor mass flow rate was measured through a calibrated I.S.A standard nozzle mounted at the entry duct and was controlled via a throttle valve. There is also a second throttling valve at the exit duct which discharges the air flow into the atmosphere through a silencer. An optical rpm meter was used to measure the instantaneous impeller speed.

An adequate data logging system was used to measure the time averaged, pressures at inlet and outlet, ducts of the compressor using accurate Honeywell piezoelectric pressure transducers (of $162 \mathrm{PC} 01 \mathrm{D}$ type). 42 pressure taps were mounted on the casing distributed from inlet to outlet of the impeller section along three rows with $120^{\circ}$ apart circumferentially. The diameter of each tap was $0.8 \mathrm{~mm}$. Arrangement of the taps is shown in Figure 2 .

The errors associated with the parameters used for evaluation of the compressor performance were obtained less than $2 \%$ for the mass flow rate, $0.5 \%$ for the pressure and $\pm 1 \mathrm{rpm}$ for the impeller rotational speed. Standard deviations based on root mean square (RMS) assumption for the flow coefficient and the loading factor were 

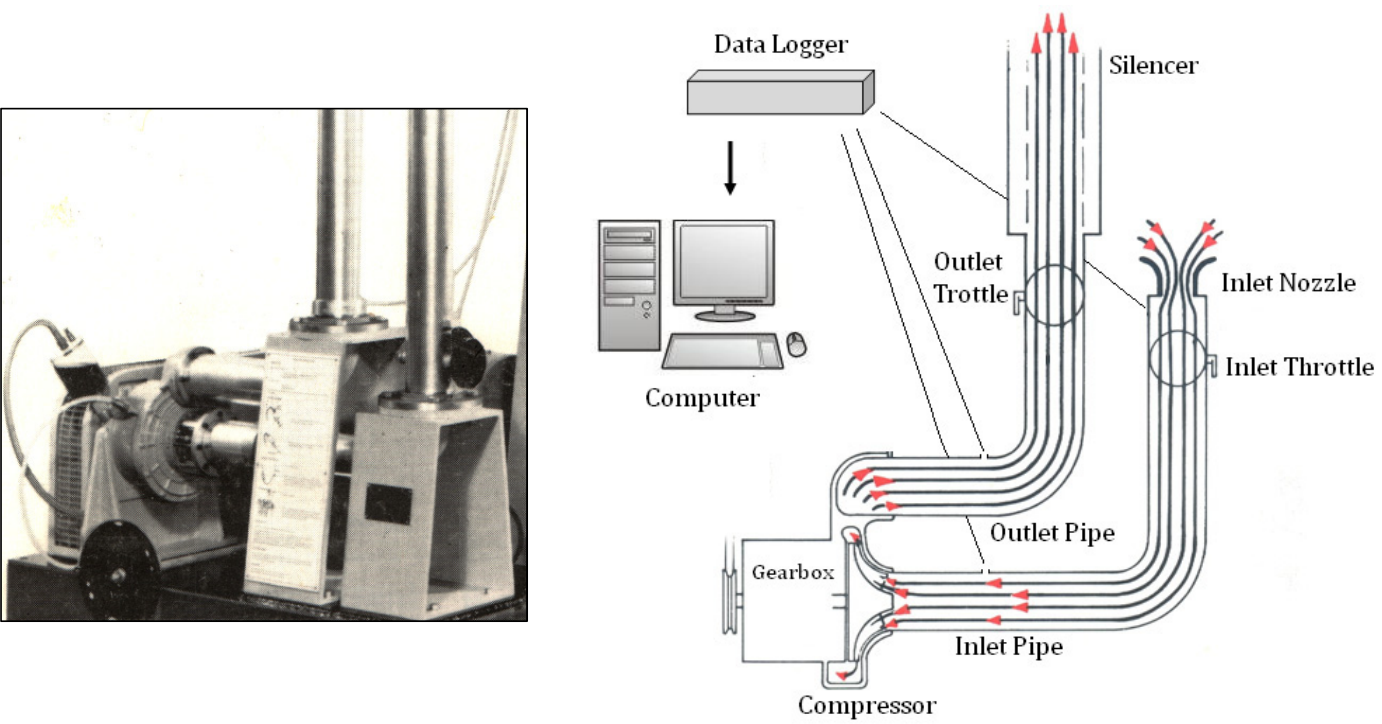

Fig. 1. IUST centrifugal compressor test rig.
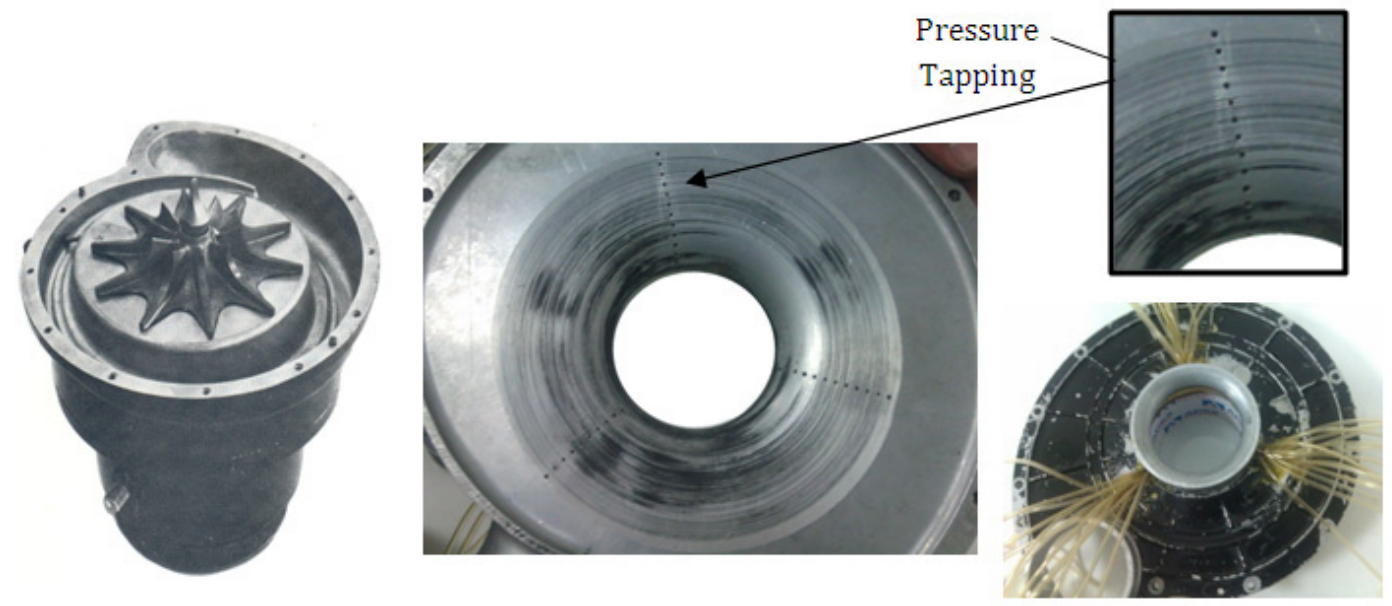

Fig. 2. IUST centrifugal compressor and casing pressure taps.

Table 1. Compressor specifications and ambient conditions.

\begin{tabular}{lc}
\hline Rotational speed $(\mathrm{rpm})$ & 8400 \\
Impeller inlet diameter $(\mathrm{mm})$ & 63 \\
Impeller outlet diameter $(\mathrm{mm})$ & 145 \\
Tip chord length $(\mathrm{mm})$ & 46 \\
Ambient pressure $(\mathrm{Pa})$ & 87500 \\
Ambient temperature $(\mathrm{K})$ & 300 \\
\hline
\end{tabular}

less than $0.2 \%$ and $0.6 \%$, respectively. Considering all the above errors and using root sum square (RSS) assumption, experimental uncertainties were estimated with less than $0.7 \%$ for the flow coefficient and $2 \%$ for the loading factor.

Numerical model which is consisted of the compressor impeller and volute is constructed through coordinate measuring machine (CMM) processing of the real compressor.

\section{Numerical approach}

Computational grid structure contained about 2550000 cells with structured hexahedral meshes; 312000 in the inlet duct; 1688000 within the impeller; 531000 in the volute and 19000 in the outlet duct. For each impeller passage, the numbers of nodes were 40, 97 and 60 in spanwise, streamwise and pitchwise directions, respectively. Twenty spanwise grid cells were generated to explore the flow phenomena inside the blade tip clearance space. The surface grid structure of this centrifugal compressor is shown in Figure 4.

In order to resolve the boundary layer effects, finer grids were used for the cells adjacent to the stationary walls and the blade solid surfaces. Density of grids close to the walls was set to keep $y^{+}$below $5 . y^{+}$is defined as the non-dimensional wall distance and is expressed as:

$$
y^{+}=\frac{u_{\tau} y}{\nu}
$$




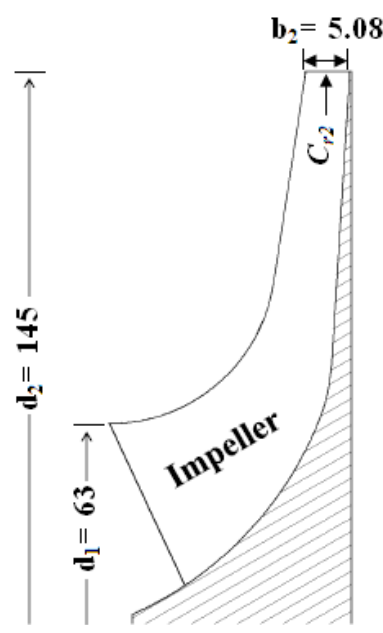

(a) Impeller dimensions (all dimensions in $\mathrm{mm}$ )

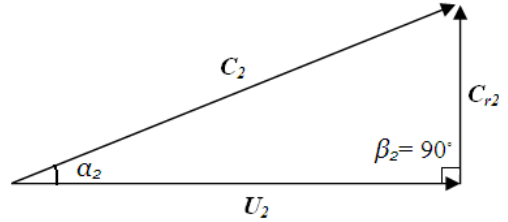

(b) Velocity triangle at the impeller exit

Fig. 3. Impeller dimensions and exit velocity triangle.

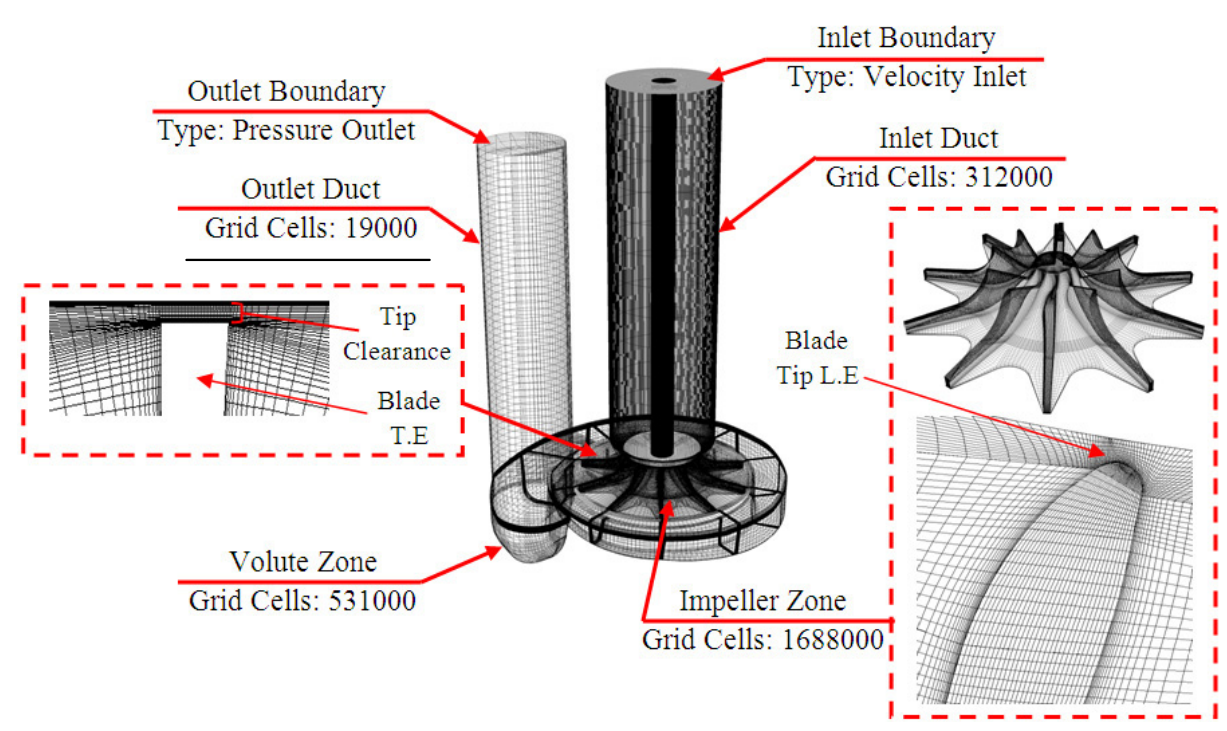

Fig. 4. Surface grids of the centrifugal compressor configuration.

where $u_{\tau}=\sqrt{\tau}_{w} / \rho$ is the friction velocity and $y$ is the normal distance from the wall at the cell center. So, it would be possible to evaluate viscosity flux close to the walls without using any wall function. In this approach, the turbulence model is modified to enable the viscosity-affected near-wall region (viscous sub-layer and buffer layer) to be resolved, properly.

The grid independency process was checked out, especially for the spanwise and pitchwise directions. In this respect, different numbers of nodes have been distributed in the spanwise (i.e., blade root to tip and the tip gap regions) and pitchwise (i.e., blade-to-blade) directions. The overall compressor performance was evaluated for different mesh structures consisted of total number of cells ranged between 324000 and 5802000 . The relative difference between the loading factor values, calculated for these two extremes, was $3.42 \%$. No considerable variations in the results have been observed for the grid numbers exceeding 2550000 .

In order to conduct the grid convergence study, loading factor $(\psi)$ was computed on three computational grid types of coarse, medium and fine, each with twice the number of grid points distributed in the spanwise and pitchwise directions, respectively. The finest one, of 2550000 cells, has been considered for the present computational task.

Grid convergence index (GCI), introduced by Roache [11], has been used as a criterion for the grid convergence studies, too. Roache suggests this index to provide a simple method for reporting the grid-convergency level. The GCI is based on generalized Richardson extrapolation involving comparison of discrete solutions at 
different grid spacings. The order of accuracy, $p$, from three solutions $(f)$ using a constant grid refinement ratio $(r)$ is determined by the following equations.

$$
p=\frac{\ln \left(\varepsilon_{32} / \varepsilon_{21}\right)}{\ln (r)}
$$

with

$$
\varepsilon_{i+1, i}=f_{i+1}-f_{i}
$$

Subscript 1 indicates the finest grid. The GCI for the fine grid solution is defined as:

$$
G C I_{i+1, i}=\frac{F_{s}\left|\varepsilon_{i+1, i}\right|}{f_{i}\left(r^{p}-1\right)}
$$

A safety factor of $F_{\mathrm{S}}=1.25$ is used here, since three grids are used in estimation of the $p$ parameter.

The GCI for grids 1 and $2\left(\mathrm{GCI}_{21}\right)$, and 2 and 3 $\left(\mathrm{GCI}_{32}\right)$ have been obtained $0.1573 \%$ and $0.4851 \%$, respectively. Reduction of GCI from the coarser grid to the finest one indicates that the solution on the finest grid resolution is nearly grid independent. Consequently, it has been decided to use a computational grid structure containing about 2550000 cells.

Steady boundary conditions were imposed at the inlet and outlet pipes of the compressor. Locations of these boundaries are shown in Figure 4. Air velocity was applied to the entrance of the inlet pipe located $0.3 \mathrm{~m}$ above the impeller leading edge with no pre-whirl. In addition, static pressure was specified uniformly at the end of the outlet pipe, $0.3 \mathrm{~m}$ downstream of the end section of the volute. Static pressure distribution was enforced on the outlet boundary based on the radial equilibrium law according to equation (5).

$$
\frac{\partial p}{\partial r}=\frac{\rho v_{\theta}^{2}}{r}
$$

No slip condition was considered on all the solid walls. Solution domain was divided into stationary and moving frames with respect to an inertial reference frame. So, the equations of motion were modified to contain Coriolis and centrifugal acceleration terms due to the transformation from the stationary to the moving zones. The governing equations are as follow:

$$
\begin{gathered}
\nabla \cdot \vec{v}_{\mathrm{r}}=0 \\
\frac{\partial\left(\rho \vec{v}_{\mathrm{r}}\right)}{\partial t}+\nabla \cdot\left(\rho \vec{v}_{\mathrm{r}} \vec{v}\right)+\rho(\vec{\Omega} \times \vec{v})=-\nabla p+\nabla \overline{\bar{\tau}}
\end{gathered}
$$

$\overline{\bar{\tau}}$ as the stress tensor, is defined by equation (8).

$$
\overline{\bar{\tau}}=\mu\left[\left(\nabla \vec{v}+\nabla \vec{v}^{T}\right)-\frac{2}{3} \nabla \cdot \vec{v} I\right]
$$

In the above equation, $\mu$ is the dynamic viscosity, $I$ is the unit tensor and the second term on the right hand is the effect of volume dilation. $\vec{\Omega} \times \vec{v}$ term appeared in the momentum equation (Eq. (7)) represents the Coriolis and centrifugal accelerations.

The well-known commercial flow solver package of Fluent has been used for the current study. The

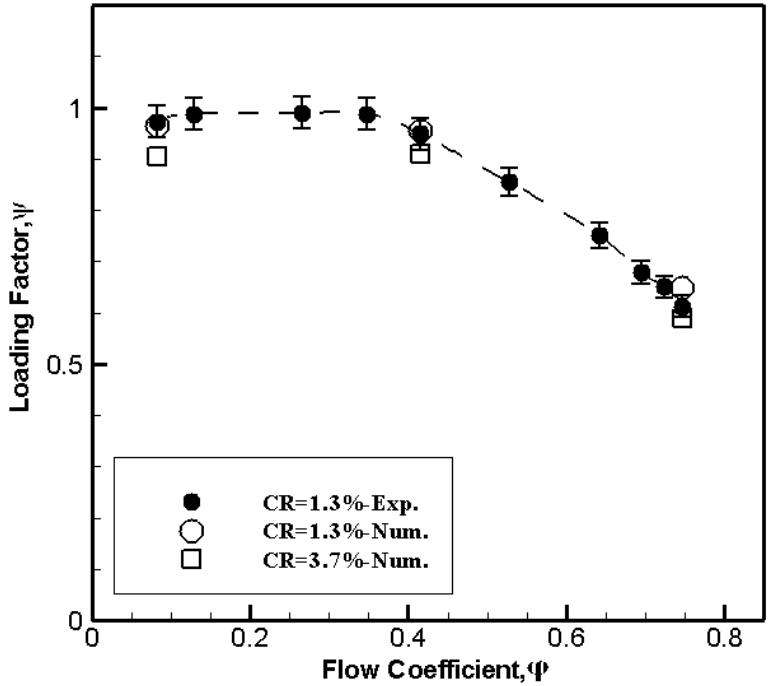

Fig. 5. Comparison between numerical and experimental performance maps, $N=8400 \mathrm{rpm}$ and $R e=6.2 \times 10^{5}$.

adopted flow solver was a three-dimensional, viscous, time-accurate code employing a segregated implicit second order scheme for solution of the Reynolds-Averaged Navier-Stokes (RANS) equations. Turbulence effects were modeled using standard $k-\varepsilon$ model. An upwind second order model was employed for discretization of $k, \varepsilon$ and momentum equations. Cell-centered finite volume scheme has been used for solution of the governing equations of continuity and momentum. Pressure-velocity coupling was performed based on SIMPLE algorithm. Time discretization was carried out using second order implicit scheme for solving the unsteady equations. At the first step of flow simulation, a steady state flow simulation has been executed to provide the initial condition for the unsteady simulation. Obviously, this technique helps in reduction of the computational time for the final convergence. The time step was chosen in such a way that the compressor impeller was rotated $0.5^{\circ}$ at every time step. As a result, a time step of $9.9 \times 10^{-6} \mathrm{~s}$ was used in the present study (which is consistent with the compressor rotational speed of $8400 \mathrm{rpm}$ ). CFL values varied between 1 and 4 . Thirty inner iterations were considered for each time step. The convergence criterion in numerical simulation was the residual values of the main governing equations approaching to around $10^{-7}$. Data monitoring of some flow variables, including pressure and velocity, as the supplementary convergence criteria, were carried out at several selected points until the final convergence was achieved.

\section{Comparison between numerical and experimental performance maps}

Three cases of "design point"; "low flow", accompanied by stall phenomenon; and "high flow" condition which is far from the stall condition, are studied. Predicted numerical results for the tested compressor with $C R=1.3 \%$ and $3.7 \%$ at three pre-defined operating 


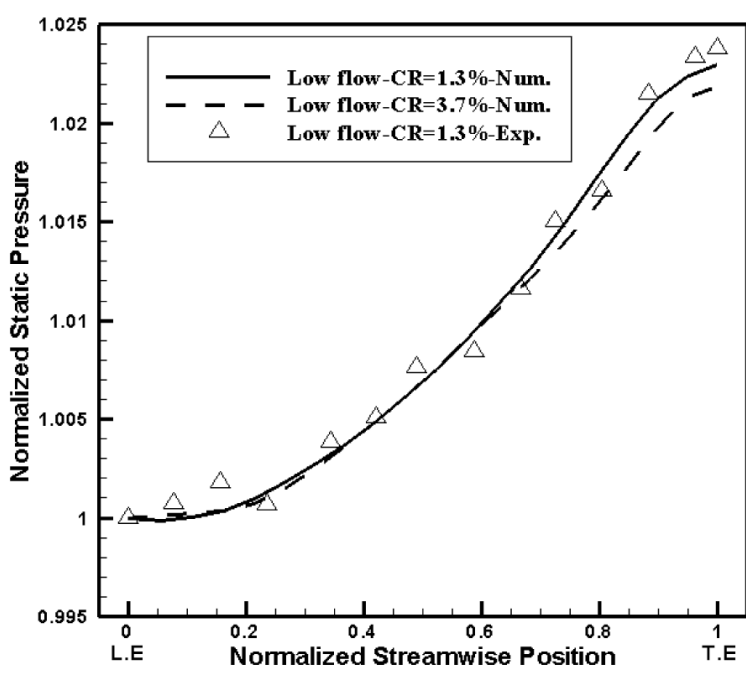

(a) Low flow

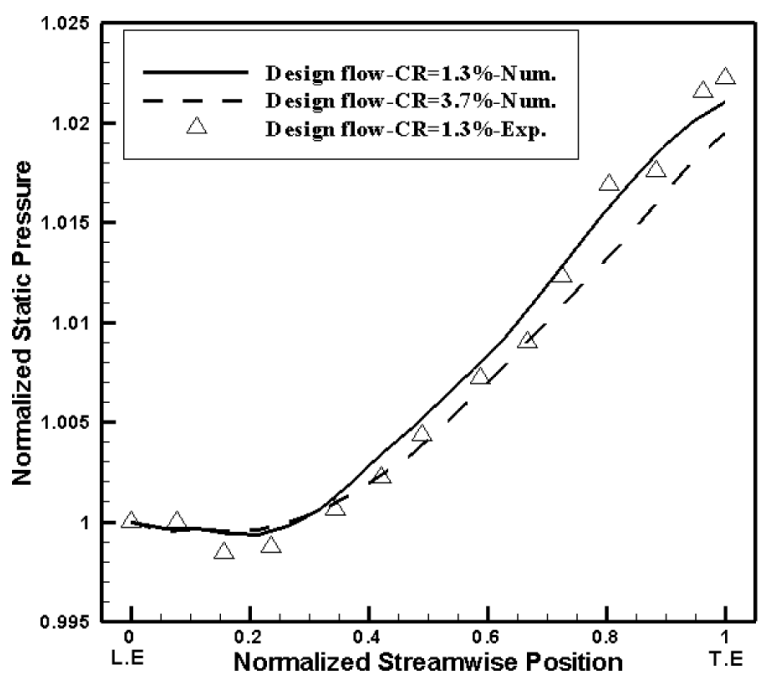

(b) Design point

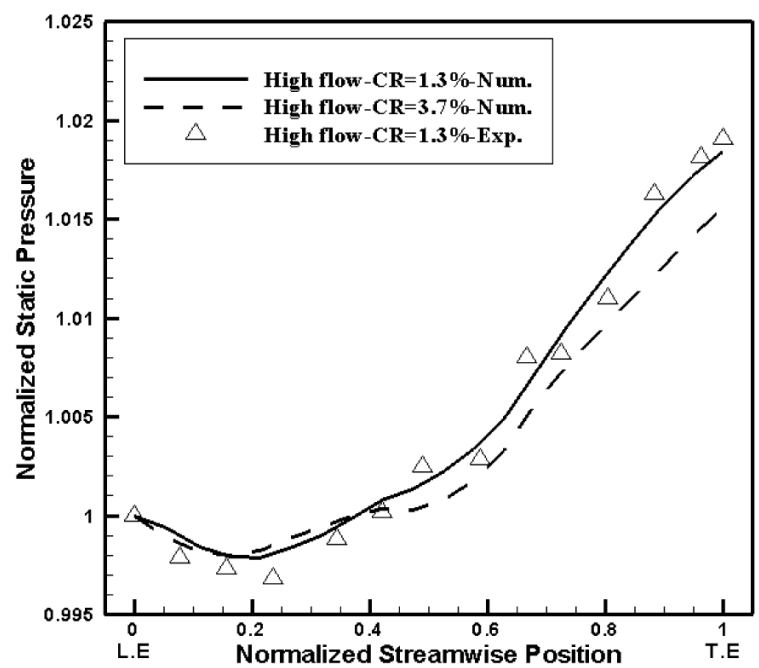

(c) High flow

Fig. 6. Normalized circumferential-averaged static pressure distribution with respect to the inflow pressure of the impeller, $N=8400 \mathrm{rpm}$ and $R e=6.2 \times 10^{5}$.

conditions are shown in Figure 5. As can be observed in this figure the numerical performance data for $C R=1.3 \%$ is in close agreement with the experimental results. Flow coefficient $(\varphi)$ and loading factor $(\psi)$, as the compressor performance parameters, are defined by the following correlations [12].

$$
\begin{aligned}
\varphi & =\frac{Q}{\pi d_{2} b_{2} U_{2}}=\frac{C_{\mathrm{r} 2}}{U_{2}} \\
\psi & =\frac{\Delta P_{\mathrm{o}}}{1 / 2 \rho U_{2}^{2}}
\end{aligned}
$$

The geometric parameters and velocity components appeared in equations (9) and (10) are introduced in Figure 3.

According to Figure 5, increasing the tip clearance ratio from $1.3 \%$ to $3.7 \%$ causes the compressor loading factor to decrease. The reason can be interpreted as follows. Interaction of the tip leakage flow and the main flow causes the leakage flow to roll up into a vortical flow configuration. Tip leakage vortices produce a low-energy fluid flow in the tip region and consequently cause a considerable blockage inside the passage. This blockage reduces the throughflow effective area and because of the mass continuity displaces the mainstream flow in the passage. So, fluid flow may turn extremely and become subjected to separation inside the passage. Increment of tip clearance causes more tip leakage flow and separation. As a result, it can be concluded that the total pressure rise across the impeller decreases while the tip clearance ratio increases [13].

\section{Flow field results}

The overall pressure rise in a centrifugal compressor is mostly dependent on the increase of pressure within its impeller. Figure 6 illustrates the numerical 

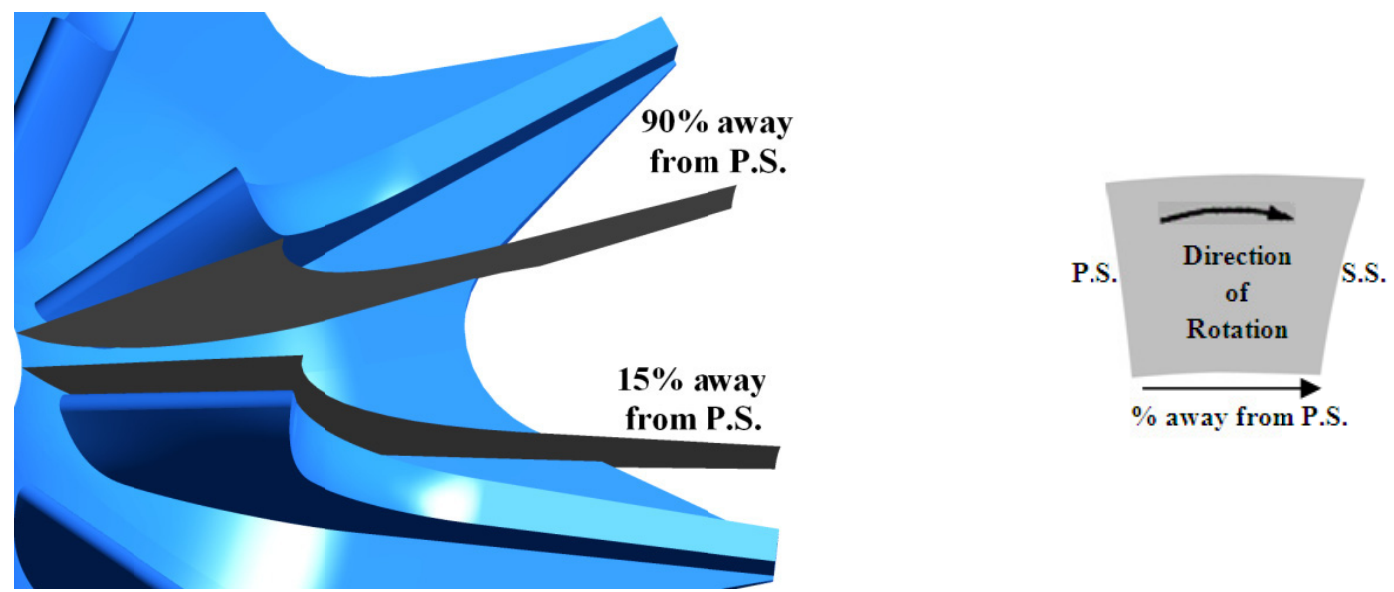

(a) Locations of meridional planes

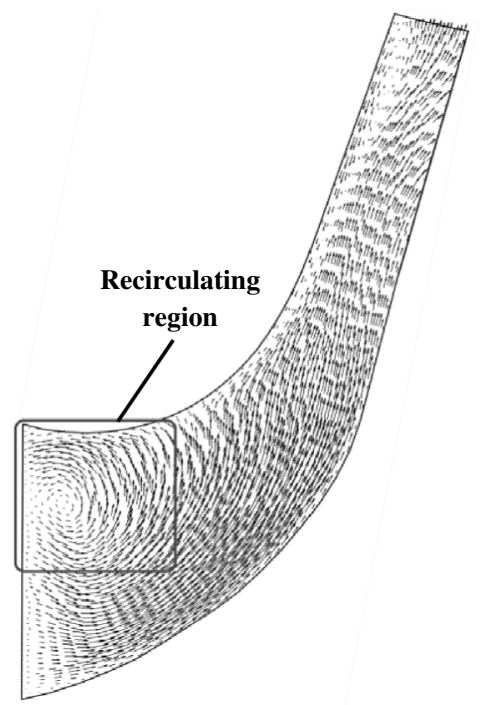

(b) Low flow $(\varphi=0.08)$

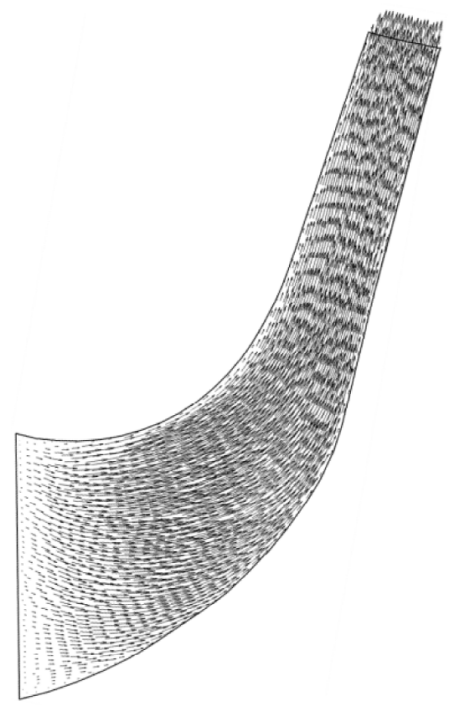

(c) Design point $(\varphi=0.41)$

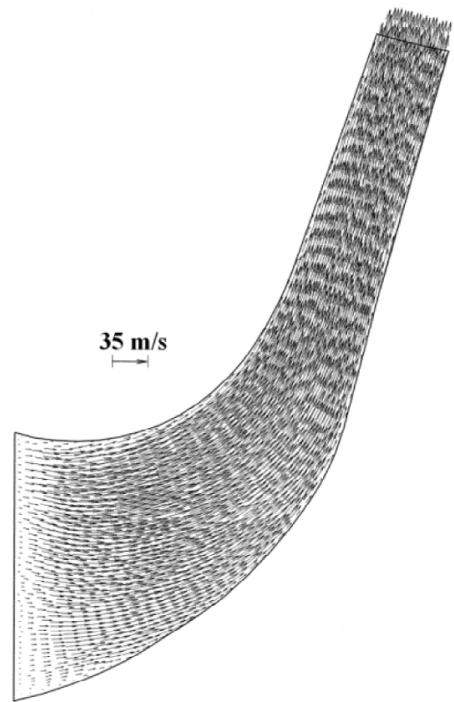

(d) High flow $(\varphi=0.74)$

Fig. 7. Meridional velocity vectors at $15 \%$ spacing from the pressure surface, $C R=1.3 \%, N=8400 \mathrm{rpm}$ and $R e=6.2 \times 10^{5}$.

and experimental results of the circumferential-averaged static pressure distribution from inlet to outlet of the impeller at three operating conditions for $C R=1.3 \%$ and $3.7 \%$. These operating conditions correspond to flow coefficients of $0.08,0.41$ and 0.74 which are referred to as low flow, design point and high flow, respectively. Good agreement can be observed between the numerical and experimental results for $C R=1.3 \%$ for all the operating conditions. Static pressure is normalized with respect to the inflow pressure of the impeller at each operating point.

According to Figure 6, static pressure rise within the impeller decreases by increasing the tip clearance size for all the operating conditions. This is in consistence with the previous observation about the loading factor $(\psi)$ of the compressor presented in Figure 5. Positive slopes can be observed in the pressure results (see Fig. 6) in the streamwise direction except to a small region aft the leading edge (between 0 to $20 \%$ chord length) for the high flow case (i.e., $\varphi=0.74$ ). At the low flow condition (i.e., $\varphi=0.08$ ), a moderate positive gradient in static pressure occurs at the impeller inlet region, in comparison to the intensive negative gradient in the inducer section of the impeller at the high flow condition. The same trends in the pressure results have also been observed by other researchers (see for example [7,14]).

Meridional velocity vectors at a $15 \%$ distance from the pressure side of the impeller for $C R=1.3 \%$ are shown in Figure 7 for different flow conditions. A circulatory region in the impeller inlet can be observed at the low flow (near stall) condition, but not at the design and high flow conditions. Decompression zone of the flow at high flow condition (as can be seen in Fig. 6c) is due to the inducer section of the impeller. In these conditions, the inducer acts as an accelerating nozzle and provides the inflow condition for the radial section of the impeller. But, at the low flow (near stall) condition, accompanied by local flow recirculation after the leading edge (Fig. 7b), the circulatory flow migrates upstream and causes the static pressure to increase at the impeller inlet region. Therefore, 

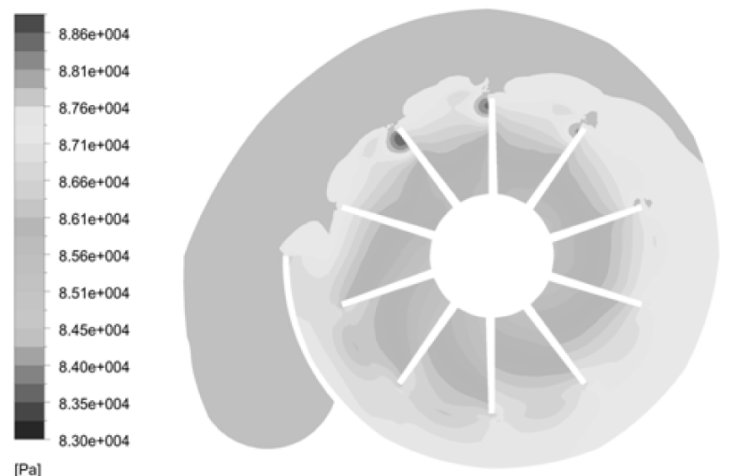

(a) Low flow

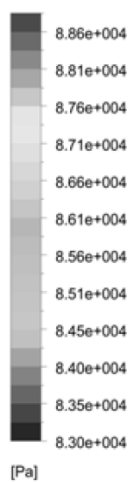

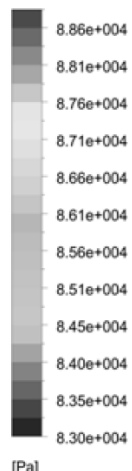

(b) Design point

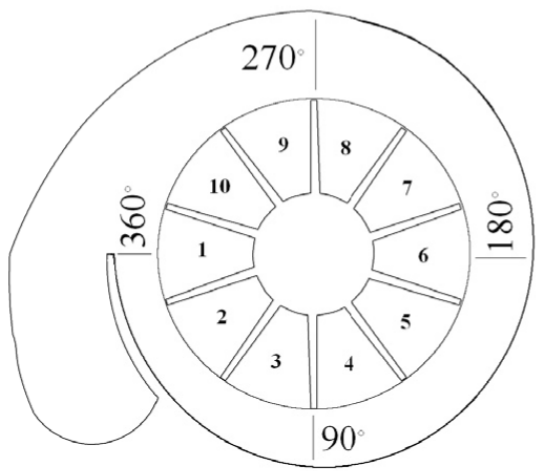

(c) High flow

Fig. 8. Static pressure distribution from impeller inlet to volute exit, $C R=1.3 \%, N=8400 \mathrm{rpm}$ and $R e=6.2 \times 10^{5}$.

at this flow condition there would not be any decompression in the inducer and the static pressure monotonously increases from the leading towards the trailing edge.

Figure 8 compares static pressure distributions at the height equal to the blade trailing edge tip height from the rotor inlet to the volute exit at $C R=1.3 \%$ for the three aforementioned flow coefficients. Comparison between different flow conditions shows that at the design point condition, a relatively more uniform pressure field exists in comparison to the off-design conditions. Generally speaking, uniformity of pressure distribution from the inlet to the outlet can result in minimization of noise and vibration levels of the compressor [15]. Similar results to those presented in Figures 7 and 8 for $C R=1.3 \%$, were obtained for $C R=3.7 \%$, but, the results are not presented here for the sake of brevity.

Figure 9 shows propagation of the mass flow rates through the blade channels at the low and design flow conditions for $C R=1.3 \%$ and $3.7 \%$. Mass flow rates are normalized with respect to the averaged mass flow rate through the all channels. Numbering system within the passage is shown in Figure 8.

As can be observed in Figure 9 mass flow is distributed more uniformly in the different passages at the design condition in comparison to the low flow condition. Generally speaking, mass flow through each blade channel should be different at the off-design condition in comparison to the design flow due to the non-uniformity of the flow within the blade passages [16]. At the design flow condition, increase of tip clearance ratio does not have any considerable effect on the mass flow through each blade channel. So, the distribution of the mass flow rate inside the passage would be similar for $C R=1.3 \%$ and $3.7 \%$ at the design condition. But, at the low flow condition, this distribution is dissimilar for different tip clearance levels. According to Figure 9a, by increasing the tip clearance ratio, less flow goes through some of the impeller passages, thus causing more blockages to the main flow. So, the main flow is deflected and causes incidence angle to increase. This condition is accompanied by occurrence of the stall phenomenon inside the impeller.

\section{Influence of tip leakage flow on the core flow}

Evolution of the tip leakage mass flow from the impeller leading edge to its trailing edge for $C R=1.3 \%$ and $3.7 \%$ at design flow condition is shown in Figure 10. It is based on the integration of the velocity component normal to the blade mean camber line over the tip clearance height. According to this figure, increment of the tip 


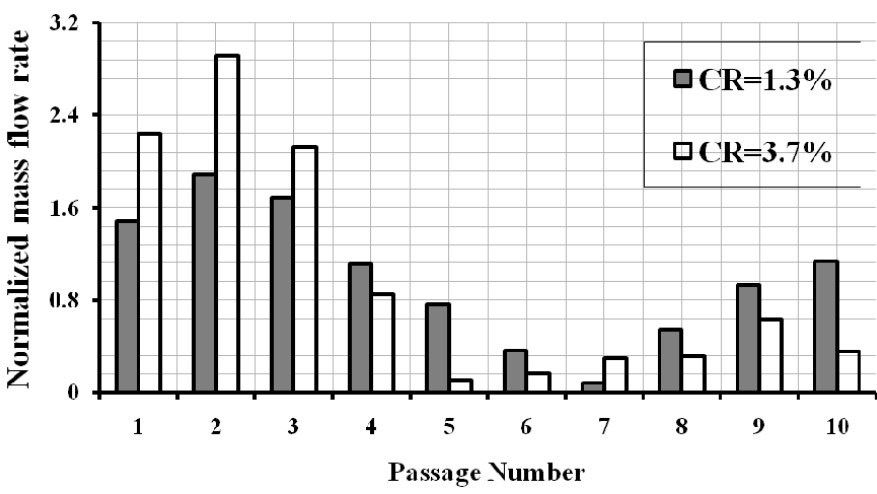

(a) Low flow

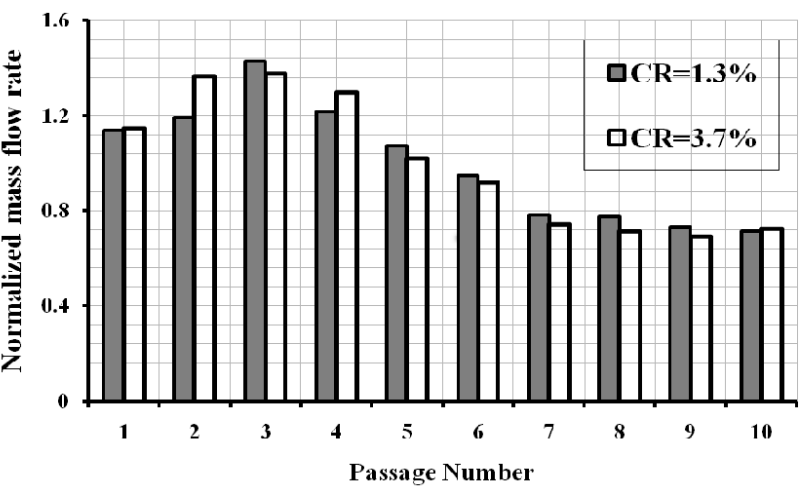

(b) Design point

Fig. 9. Normalized mass flow rate (with respect to the averaged mass flow through all channels) in each blade channel, $N=8400 \mathrm{rpm}$ and $R e=6.2 \times 10^{5}$.

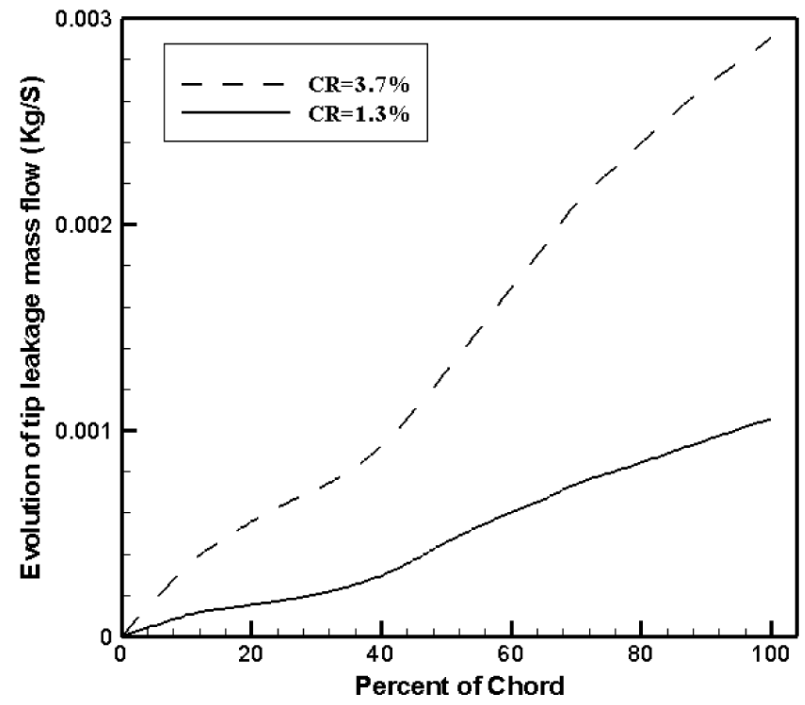

Fig. 10. Evolution of the tip leakage mass flow from L.E. to T.E. at design flow condition, $N=8400 \mathrm{rpm}$ and $R e=$ $6.2 \times 10^{5}$.

clearance ratio from $1.3 \%$ to $3.7 \%$ causes the tip leakage mass flow to increase by 1.74 times. It results in more blockages inside the impeller passages, which have already been mentioned while dealing with Figure 9.

Figure 11 compares meridional velocity vectors in the three flow conditions on a meridional surface with $90 \%$ distance away from the pressure side of the impeller for $C R=1.3 \%$. Increasing the flow coefficient has weakened the tip leakage flow. This flow is pushed downstream of the impeller due to the strong main passage flow in a way that, at high flow condition, the wake region vanishes (see Fig. 11c).

At the low flow condition, deterioration of throughflow velocity can be observed while being compared with the higher flow case. In this condition, flow at the exit of the impeller (entrance of the volute) is reversed (see Fig. 11a).
This reversed flow is accompanied by occurrence of the stall phenomenon within the volute. This event will be discussed later in the following section.

\section{Flow pattern within the volute}

Figures 12 to 15 present the swirl velocity vectors of different volute cross sections at various flow conditions at $90^{\circ}, 180^{\circ}$ and $270^{\circ}$ azimuthal angles for two different clearance levels. These angular positions have already been introduced in Figure 8. For the sake of brevity, only the results of $90^{\circ}$ azimuthal angle are presented for $C R=3.7 \%$.

Spanwise area-averaged radial velocity $\left(V_{\mathrm{r}}\right)$ at the volute inlet along the circumferential angle is shown in Figure 16 for $C R=1.3 \%$ and $3.7 \%$. $V_{\mathrm{r}}$ is normalized with respect to the peripheral velocity at the impeller exit, $U_{2}$. As can be expected, radial velocity is increased by increasing of the tip clearance level. The flow structure inside the volute is strongly dependent on the vortex flow which forms within it. Various reasons are reported to be responsible for the vortex flow formation inside the volute $[9,10]$. The presence of free or forced vortex inside the volute is dependent on its geometry and operating conditions of the compressor.

Generally speaking, at the volute entrance zone close to the compressor tongue, flow starts swirling around the center of the volute cross section. Velocity gradient and shear stress increase by this swirling action. Swirl kinetic energy is dissipated in the center of the volute until a forced vortex forms. So, vortex formation at small circumferential angles could be as a consequence of the shear stresses inside the channel. But, at the larger circumferential angles, the cause of a vortex formation at various flow conditions may be different.

As is shown in Figure 16, at the low flow condition, radial velocity in the circumferential direction is approximately constant for different clearance levels, and therefore, does not have considerable role in the vortex 


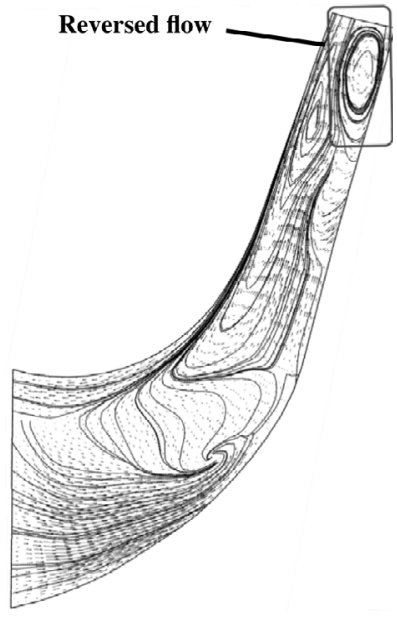

(a) Low flow

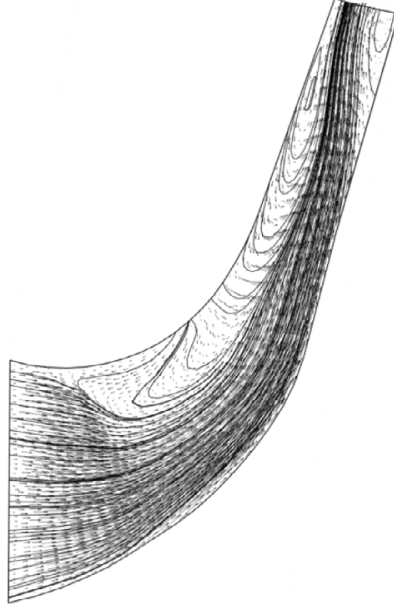

(b) Design point

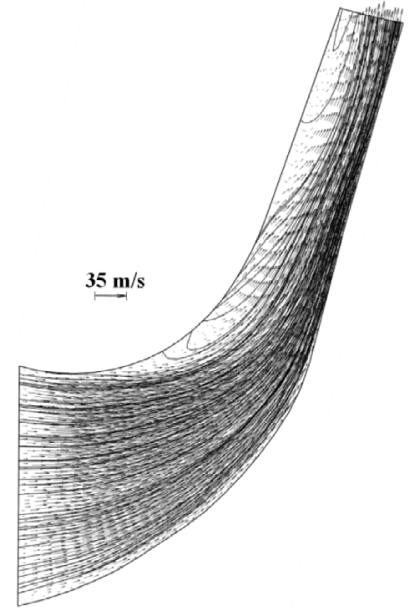

(c) High flow

Fig. 11. Meridional velocity vectors in various flow conditions at $90 \%$ distance away from P.S., $C R=1.3 \%, N=8400 \mathrm{rpm}$ and $R e=6.2 \times 10^{5}$.

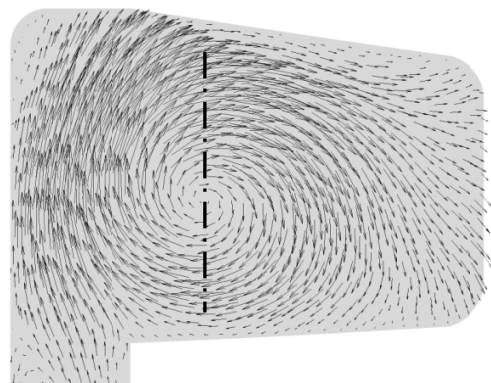

(a) Low flow

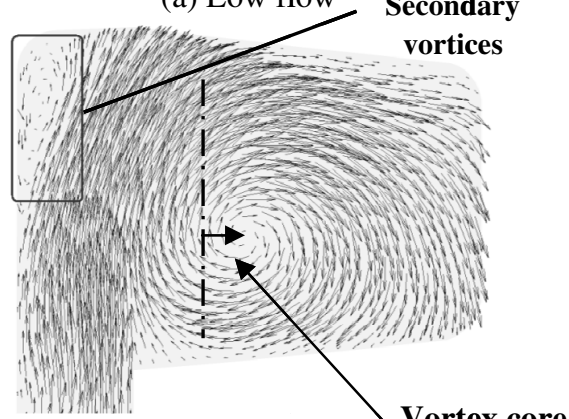

(b) Design point $>$ shift

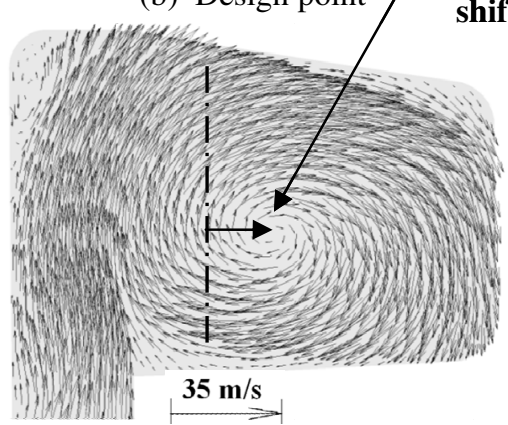

(c) High flow

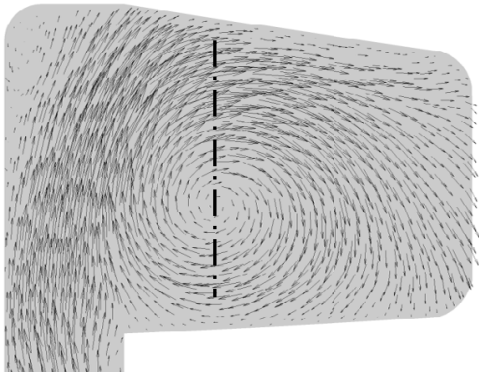

(a) Low flow

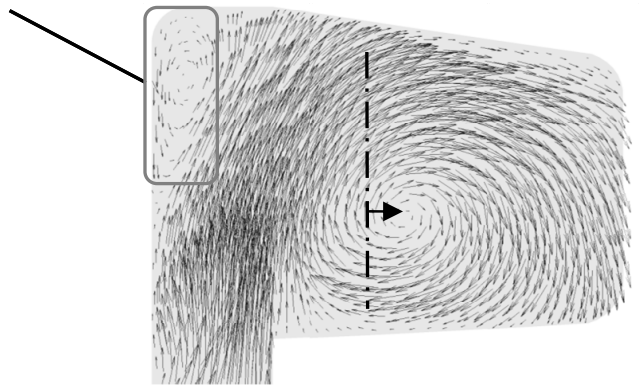

(b) Design point

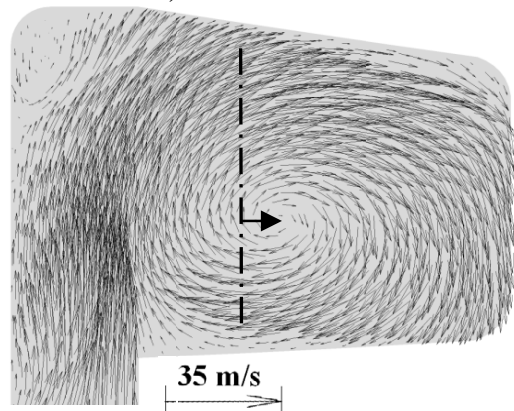

(c) High flow
Fig. 12. Swirl velocity at $\theta=90^{\circ}, C R=$ $1.3 \%, N=8400 \mathrm{rpm}, R e=6.2 \times 10^{5}$.
Fig. 13. Swirl velocity at $\theta=90^{\circ}, C R=3.7 \%$, $N=8400 \mathrm{rpm}, R e=6.2 \times 10^{5}$. 


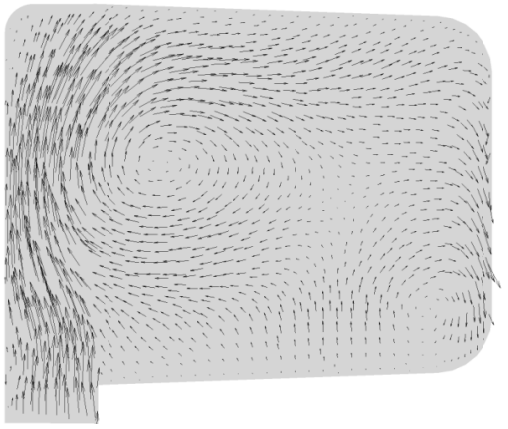

(a) Low flow

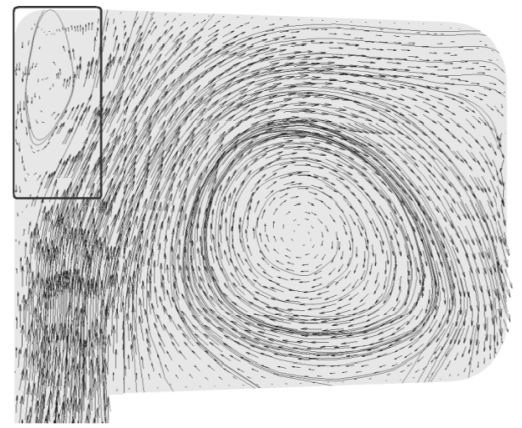

(b) Design point

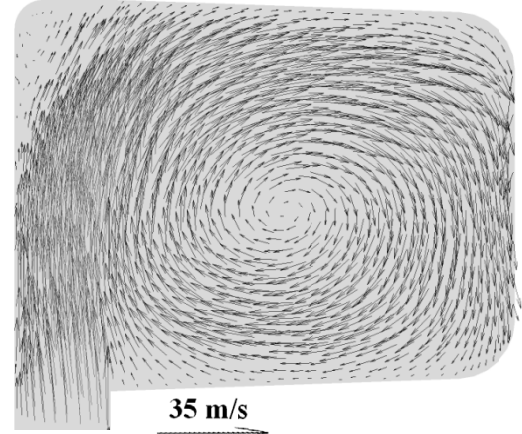

(c) High flow

Fig. 14. Swirl velocity at $\theta=$ $180^{\circ}, \quad C R=1.3 \%, N=$ $8400 \mathrm{rpm}, R e=6.2 \times 10^{5}$.

formation. So, shear stress is mainly responsible for the vortex appearing at the low flow condition. At the high flow condition and at the larger circumferential angles, radial velocity increases with the circumferential angle and causes swirl velocity to increase. So, a forced vortex is formed in the center of the volute cross section. Hence, at high flow and in the larger circumferential angles, vortex flow could be formed due to the velocity distribution in the entrance of the volute and not to the existence of the shear stresses inside the channel [9].

At the low flow (near stall) condition, and in the downstream regions of the volute, as a result of the flow separation inside the impeller, flow inside the volute begins to stall. So, occurrence of the stall inside the impeller causes the other compressor components to be stall-prone.

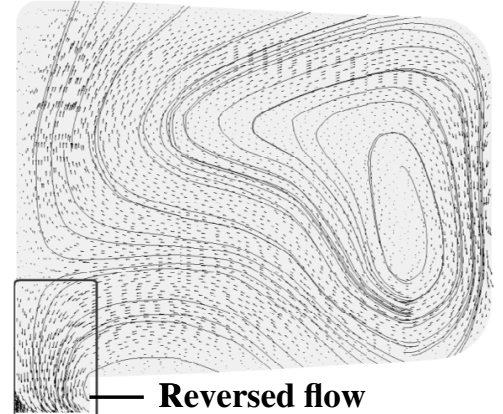

(a) Low flow

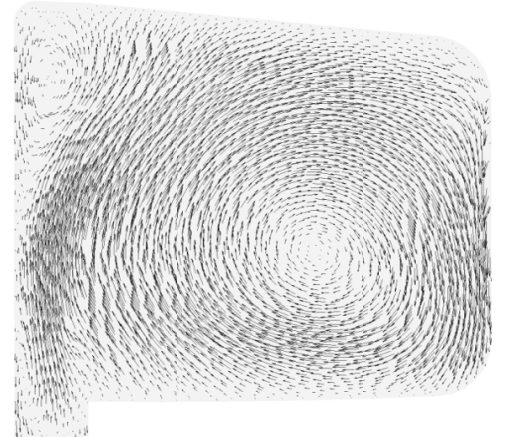

(b) Design point

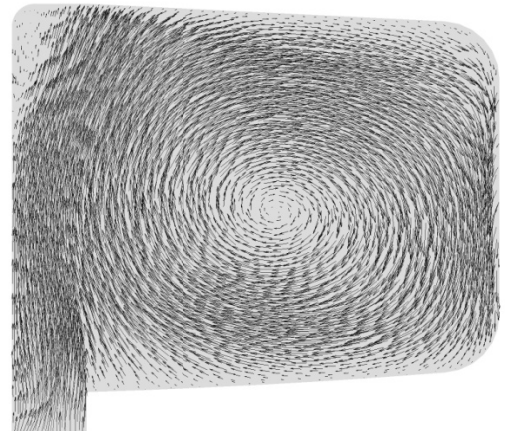

(c) High flow

Fig. 15. Swirl velocity at $\theta=$ $270^{\circ}, \quad C R=1.3 \%, N=$ $8400 \mathrm{rpm}, R e=6.2 \times 10^{5}$.

In this situation, the main volute vortex becomes weaker (see Fig. 15a). Occurrence of the stall inside the volute causes a recirculatory flow to form at the volute entrance, which in turn affects the vortex structure. Focusing on Figures 12 to 15 following observations can be concluded.

a) At any flow coefficient, the position of the vortex center changes with the circumferential angle. In addition, by increasing the mass flow rate, swirl velocity increases, thus, the vortex core can be shifted in each section towards the right wall of the volute for $C R=1.3 \%$ and $3.7 \%$ (Figs. 12 and 13 ). In addition, at any flow coefficient, by increase of the tip clearance ratio, the vortex center shifts towards the right wall. 


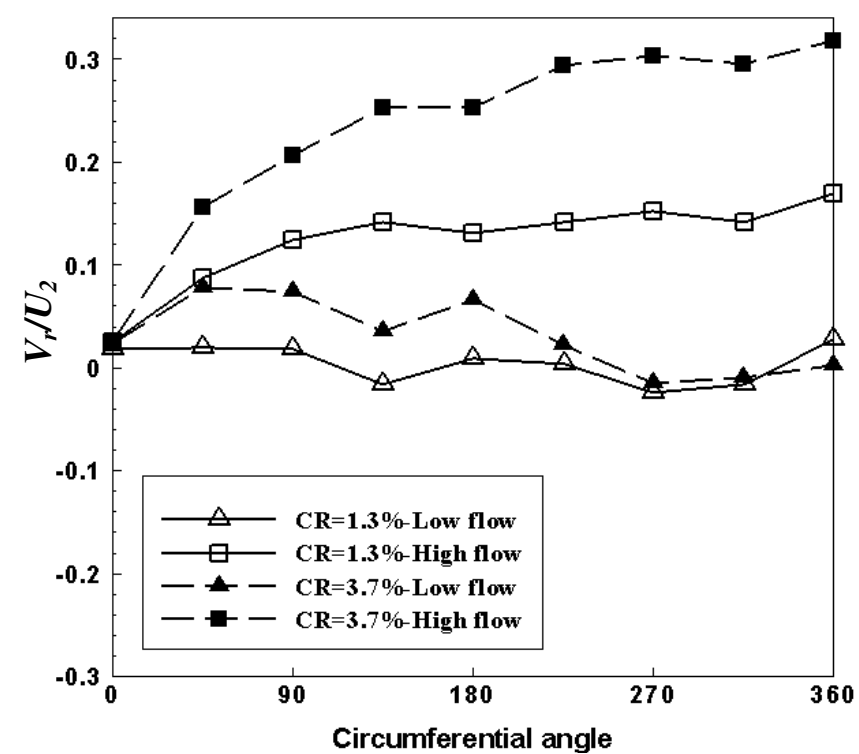

Fig. 16. Spanwise normalized area-averaged radial velocity at volute inlet $\left(V_{\mathrm{r}}\right)$ with respect to peripheral velocity at the impeller exit $\left(U_{2}\right), N=8400 \mathrm{rpm}$ and $R e=6.2 \times 10^{5}$.

b) Because of rectangular cross-section of the volute, the velocity gradient could be large and thereafter may become moderate from center of vortex to some radial distance. Whereas, in the circular or elliptical volutes, such structures may not be observed $[17,18]$.

c) A well-designed geometry of the volute causes the flow to be guided correctly, except to the top of the left wall. In this region (similar to other rectangular volutes), a secondary vortex (as can be seen in Figs. 12b and 13b) may be formed. These secondary vortices can be clearly observed in the design flow condition rather than in the off-design conditions and grow with the circumferential angle. Increase of the tip clearance ratio causes the secondary vortices to increase. The main reason of the secondary vortex formation is due to the radial pressure gradient. Although the low energy fluid inside the volute boundary layer is exposed to the above mentioned radial pressure gradient, but, it cannot follow the main swirl direction and therefore it turns back towards the lower pressure. So, the corner vortex structure may form. Flow reversing and adverse pressure gradient at the low flow and strong throughflow velocity at the high flow condition cause near-corner vortices to get weaker.

\section{Conclusions}

This paper is attributed to numerical simulation of a centrifugal compressor using RANS technique with $k$ - $\varepsilon$ turbulence modeling at different tip clearance ratios and various flow conditions attributed to near and far from the stall phenomenon. In addition to the flow field studies within the impeller, a detailed study of secondary flow structures in the other components of the compressor has been performed. The performance curve of the compressor stage is determined numerically and experimentally which shows good agreements. For a more in-depth assessment of this compressor three flow conditions, namely, high flow and design point (which are far from stall) and low flow (which is near stall condition) are examined.

The main conclusions obtained from the present research work can be outlined as follows.

(1) In far stall conditions, specifically at design flow, more uniform flow structures can be observed in the all compressor components. But at near stall condition, flow structures within the compressor deteriorate and stall affects all components, simultaneously.

(2) By increasing the impeller tip clearance ratio, tip leakage flow can be strengthened. The more strength of tip leakage flow causes the more blockage of the passage and reduction of the compressor loading factor. As the mass flow rate in the compressor decreases, adverse effects of the tip leakage flow either on the compressor performance or the stall commencement get stronger. So, increment of the tip clearance ratio increases the probability of stall occurrence inside the impeller.

(3) In case of the impeller stall, other compressor components, especially its volute, become more stall-prone. In this condition, structure of the vortex formed in the volute channel becomes weaker.

\section{References}

[1] W. Huang, S. Geng, J. Zhu1, H. Zhang, Numerical simulation of rotating stall in a centrifugal compressor with vaned diffuser, J. Thermal Sci. 16 (2007) 115-120

[2] M. Schleer, S.J. Song, R. Abhari, Clearance effects on the onset of instability in a centrifugal compressor, J. Turbomach. 130 (2008) 031002-1-11

[3] A.S. Hassan, Stability of a low-speed centrifugal compressor with casing treatments, In: Skibin V.A. Saren, V.E. Savin N.M., et al. (eds.) Turbomachines: aeroelasticity, aeroacoustics and unsteady aerodynamics, TORUS Press, Moscow, Russia, 2006, pp. 406-420

[4] H.G. Zhang, W.L. Chu, Y.H. Wu, Numerical investigation of the circumferential grooved casing treatment as well as analyzing the mechanism of improve stall margin, Proceedings of Fifth International Conference on Fluid Mechanics, Shanghai, China, 2007, pp. 448-451

[5] S. Kang, C. Hirsch, Numerical simulation and theoretical analysis of the $3 \mathrm{~d}$ viscous flow in centrifugal impellers, Task Quarterly 5 (2001) 433-458

[6] H. Chen, S. Guo, X. Zhu, Z. Du, S. Zhao, Numerical simulations of onset of volute stall inside a centrifugal compressor, Proceedings of ASME Turbo Expo 2008: Power for Land, Sea and Air, Berlin, Germany, 2008, No. GT2008-50036

[7] C. Hah, H. Krain, Secondary flows and vortex motion in a high-efficiency backswept impeller at design and offdesign conditions, J. Turbomach. 112 (1990) 7-13

[8] R.M. Chriss, M.D. Hathaway, J.R. Wood, Experimental and computational results from the nasa lewis low-speed centrifugal impeller at design and part-flow conditions, J. Turbomach. 118 (1996) 55-65 
[9] A. Reunanen, Experimental and numerical analysis of different volutes in a centrifugal compressor. Ph.D. Thesis, Lappeenranta University of Technology, 2001

[10] E. Ayder, R. Van Den Braembussche, J.J. Brasz, Experimental and theoretical analysis of the flow in a centrifugal compressor volute, J. Turbomach. 115 (1993) $582-589$

[11] P.J. Roache, Quantification of uncertainty in computational fluid dynamics, Ann. Rev. Fluid Mech. 29 (1997) 123-160

[12] S.M. Yahya, Turbines compressors and fans, Tata McGraw-Hill Publishing Company Limited, New Delhi, India, 2003

[13] M. Furukawa, M. Inoue, K. Saiki, Y. Yamada, The Role of tip leakage vortex breakdown in compressor rotor aerodynamics, J. Turbomach. 121 (1999) 469-480
[14] S. Colantuoni, A. Colella, Aerodesign and performance analysis of a radial transonic impeller for a 9:1 pressure ratio compressor, J. Turbomach. 115 (1993) 573-581

[15] N.A. Cumpsty, Compressor aerodynamics, Longman Scientific, London, 1999

[16] M. Nili-Ahmadabadi, A. Hajilouy-Benisi, M. Durali, F. Ghadak, Investigation of a centrifugal compressor and study of the area ratio and tip clearance effects on performance, J. Thermal Sci. 17 (2008) 314-323

[17] C. Xu, M. Muller, Development and design of a centrifugal compressor volute, Int. J. Rotating Mach. 3 (2005) 190-196

[18] E. Ayder, R. Van den Braembussche, Numerical analysis of the three-dimensional swirling flow in centrifugal compressor volutes, J. Turbomach. 116 (1994) 462-468 